\title{
Electric Energy Consumer Characterization, Classification and Demand Forecasting using Convolutional Neural Networks
}

\author{
Ignacio Aguirre ${ }^{1}$, Stepan V Ulyanin ${ }^{2}$, Jose Vazquez-Canteli ${ }^{2}$, Zoltan Nagy $^{2}$ \\ ${ }^{1}$ Universidad Pontificia de Comillas ICAI, Madrid, Spain \\ ${ }^{2}$ University of Texas at Austin, TX USA
}

\begin{abstract}
This paper presents a procedure to support the retail and distribution companies on the extraction of knowledge from electricity consumption data. Our final objective is to forecast individual load profiles of consumers for a particular season of the year using the historical information gathered during the months of the previous season. The algorithm classifies consumers in one of the 7 clusters, with $70 \%$ accuracy in the best case, which gives retail companies an excellent point to start in tariff customization. We encode the power use (time series) in GAFs and MTFs to represent the images that are processed by the CNNs to perform classification tasks.
\end{abstract}

\section{Introduction}

Electric utilities are currently experiencing an age of changes that are transforming the traditional business of the power industry. One of these changes is the extensive amount of data that they have but do not know how to manage in a useful manner to reduce consumer costs and improve the overall system efficiency. This paper presents a procedure to support the retail and distribution companies on the extraction of knowledge from electricity consumption data. One of the significant consequences of the electricity markets liberalization is the freedom that all customers will have in choosing their particular electricity supplier. This open market creates an environment where retail companies compete for the electricity supply of end users. The knowledge of how and when consumers consume electricity is the key to be competitive in the electric retail industry Figueiredo et al. (2005). From historical data of different consumer demand, it is possible to derive this kind of knowledge. It is essential to classify every consumer in one of the classes represented by its load profiles. The number of classes in which we separate the data is an input that we must introduce when assigning the labels to consumers, and it must be based on the level of compactness needed and the results obtained in the forecasts.

Once we characterize new consumers, there is a better chance to offer tariffs that will benefit them in the long term. Furthermore, this characterization provides several advantages:

1. Accurate load forecasting for better system expansion planning to achieve good service quality Figueiredo et al. (2003)

2. It might help to reduce the costs associated with an excess of power capacity in the design of electric equipment required for distribution such as lines and transformers Mill (2016).

3. It might help retail companies to determine which consumers will be benefited by Demand Respond Programs such as Real Time Pricing tariffs Albadi and El-Saadany (2007) Valero et al. (2004).

This paper bases on the application of clustering techniques for the determination and characterization of a set of load profiles, representing the different consumption patterns of a sample of consumers. The final objective is to forecast individual load patterns for a season of the year using the information gathered during the previous season. To achieve this, we use Convolutional Neural Networks and K-means algorithm to link for example spring inputs of consumers with their summer clustering classes. Zhiguang and Tim (2015) shows the techniques to encode time series as images that we can directly apply to our data. In his conference paper, he proposes a framework to encode time series as different types of images, namely, Gramian Angular Fields (GAF) and Markov Transition Fields (MTF). The definition of these fields allows the use of Convolutional Neural Networks as the algorithm to classify new consumers to the specified classes. The prediction of the behavior of new consumers one season ahead will help retail companies in personalizing electric tariffs and in planning the amount of electricity they need to buy. The reduction of the unpredictability associated with electrical consumption will lead electricity providers to reduce costs. These reductions in costs must affect consumers in the way of savings in their electricity bills. 
The paper is organized as follows: in the first part, the characterization section explains the methodology to prepare and transform the data. The forecasting section explains the conversion process of time series into MTFs and GAFs. Then, we present a case study with real data to analyze the performance of our method. Finally, the results and conclusions of the work will close the document.

\section{Methodology}

For this work, we developed a procedure to reduce the uncertainty in residential electricity demand to help electricity providers in offering personalized electric tariffs to new clients. Figure 1 depicts this process using a block diagram to show the procedure flow. The first step is to carry out a characterization study of the different classes of electric behaviors among the consumers. Once we define the classes, it is possible to customize electric tariffs that will adapt to each of the classes defined. A good approach with new consumers would be to initially offer them a fixed price tariff until electricity providers gather enough information about their consumption patterns. After collecting information for the first month, we can classify consumers in one of the classes defined by the clustering. At this point we will compare the performance of the CNNs model and the K-means algorithm in the classification task. Having consumers classified, it is possible to offer them the tariff that will better fit their needs. Once we collect one season of data, it is possible to forecast in which cluster class the client will be the next season. Classifying consumers for the next season is a more difficult task because the algorithms now must classify consumers using information from a different period. For the forecasting, instead of using three months of data averaged to construct a representative daily profile, the data will be averaged per month with the purpose of losing the least information possible. But after doing some simulations, it can be stated that the data that have more predicting power is the most recent one to the season that wants to be predicted. An explanation of this fact is that recent data will be more similar in shape to the profiles that we pretend predict. For this reason, we decided to use just the third month of the previous season to predict consumer classes of the next season. Therefore, electric tariffs will be reviewed once every three months. Our case study will focus on the seasons of spring and summer which give the best results. More results concerning the rest of the year will also be provided at the simulation and results section.

The characterization section summarizes the steps to prepare the data to obtain the different classes of customers. It explains which parameters are the key to achieve an accurate generalization of

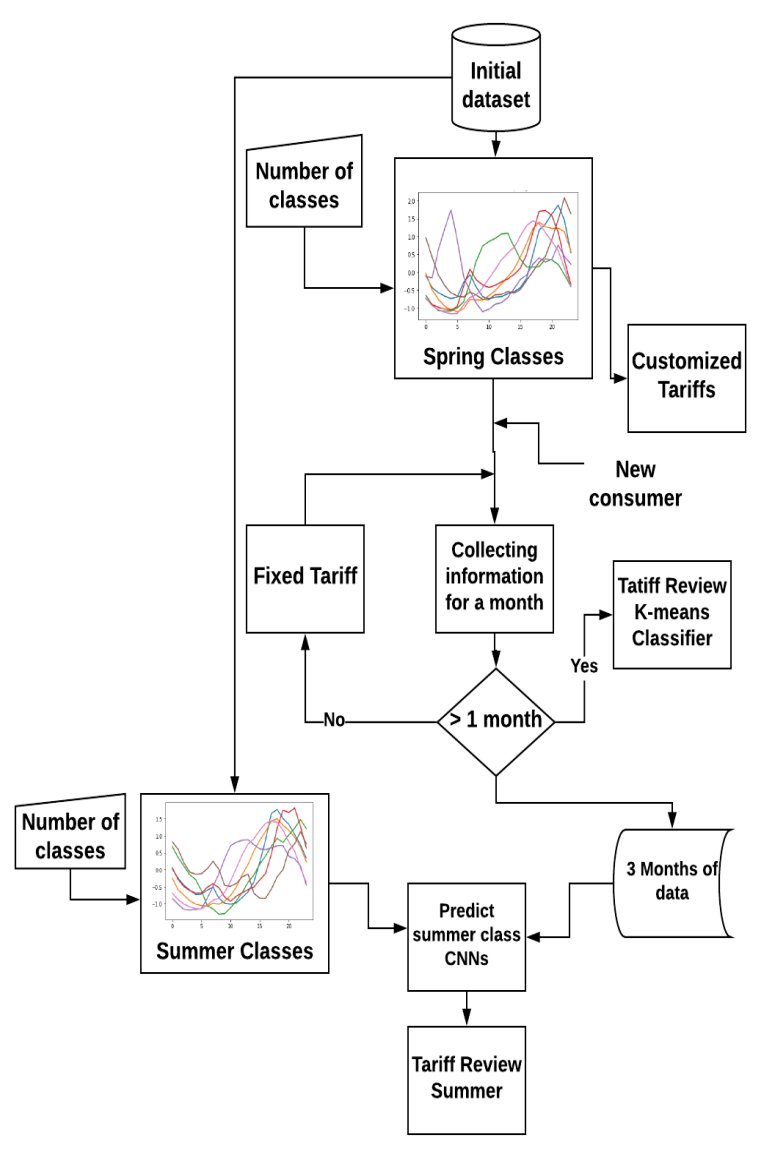

Figure 1: Classification procedure.

the typical consumers presented in our data. The forecasting section summarizes how to encode time series as images that the CNN model uses as inputs. This section explains why Markov Transition Fields and Gramian Angular Fields can be combined as 3 channels to form an image and why this combination is effective in predicting consumer patterns.

\section{Characterization section}

The characterization section is organized in the following steps:

Data selection and cleaning: the first step is the selection of the data with significance to the process. It is necessary to conduct different studies depending on the voltage level of consumers. In this case, this work focuses on the residential level. The power use is measured as the total amount of power consumed by a home in an instant, and therefore it includes the sum of all the different electrical devices and appliances of a house. In the cleaning phase, we look for inconsistencies in the data and we remove consumers with null elements from the initial dataset.

Data reduction: the season of the year and the type of day (working days or weekends) affect electricity 


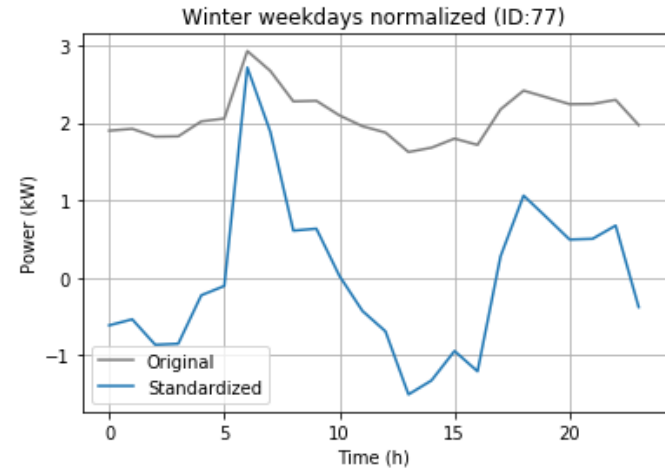

Figure 2: Comparison of the original and the normalized winter weekdays representative load diagram for ID $7 \%$.

consumption patterns. We use these characteristics to separate the data into smaller datasets. Two datasets represent each season of the year, one for working days and another for weekends. The consumer data of each season of the year is reduced to one daily load profile which is known as the representative load profile. Representative load profiles are obtained by averaging the load profiles of the whole season to one day. The data is averaged along its dimensions which are the 24 hours of a day. Each consumer is then described by one single representative load profile in each dataset, for the different loading conditions. For example, Consumer $\mathrm{X}$ will have a representative daily load diagram for winter weekdays and a different one for summer weekdays.

Data normalization: we construct the load profiles using the field-measurement values, so they need to be brought together to a similar scale for their comparison. The representative load diagram of each consumer was normalized using Z-normalization. This procedure ensures that all elements of the input vector are transformed into the output vector whose mean is approximately 0 while the standard deviation is in a range close to 1 . This kind of normalization allows maintaining the shape of the curve and comparing the consumption patterns. Figure 2 illustrates the differences between the raw and Z-normalized time series. The normalization formula is shown below:

$$
x_{i}^{\prime}=\frac{x_{i}-\mu}{\sigma}
$$

Load profiling: here the goal is the partition of the data sample into a set of classes defined according to the load shape of the representative load diagrams of each consumer. The data-mining model for consumer characterization is based on the unsupervised learning technique clustering K-means to form the clusters. The representative load diagram of the $\mathrm{m}^{\text {th }}$ consumer is the vector $p^{(m)}=\left\{p_{1}^{m}, \ldots, p_{h}^{m}, \ldots, p_{H}^{m}\right\}$ where $p_{h}^{m}$ describes the normalized values of the power consumed at the instant $\mathrm{h}$ and $h=1, \ldots, H$ with $H=24$ or $H=96$ depending of the use of 1-hour interval or 15-min interval resolution. The use of 1-hour interval or 15-min will depend on the application of the classification. K-means algorithm compares the power use of all consumer at each of the 96 dimensions to minimize the distance between elements in the same cluster. The centroids represent the average of all those elements grouped in the same cluster. K-means algorithm would classify the same two time series in two different classes if they are lagged, as it considers every dimension independently (every hour, every $15 \mathrm{~min}$ ). 15-min interval time series are more sensitive to this issue and thereby are a worse option if the objective is to find the class that shares similar metadata characteristics, such as, the size of houses or the number of members in a house. On the other hand, CNNs take into account temporal relationships between dimensions (this information is provided by GAFs and MTFs) in a similar way as an ACF does with time series. 15-min interval time series are more precise for studying the simultaneity between consumers and tariff designing. They also have a better performance on CNNs and this is why they are our final choice.

The classes obtained in the clustering represent the different consumption patterns among the sample in the study. The load profiles of the centroids represent each of these classes. A good consumer characterization will have a great impact on the success of the forecasting module. A functional characterization must ensure well-separated classes (a proper distinction between class representative load diagrams) and compacted classes (the load diagrams included in each class must be similar to their representative centroids). To evaluate the performance of the algorithm we use two measures of adequacy: a measure of cluster compactness (MIA) and another measure of cluster separation (CDI). Chicco et al. (2003) presented both measures in his work. Inputs with smaller MIA and CDI values are considered to have sharper discriminating properties. Considering a set of $\mathrm{X}$ load diagrams separated in $\mathrm{K}$ classes with $\mathrm{k}=1, \ldots, \mathrm{K}$ and each class is formed by a subset $C^{(k)}$ of load diagrams, where $r^{(k)}$ is a pattern assigned to cluster $\mathrm{k}$ (cluster centroid), Chicco et al. (2003) defined the following performance measures:

a) Mean Index Adequacy (MIA):

$$
M I A=\sqrt{\frac{1}{K} \sum_{k=1}^{K} d^{2}\left(r^{(k)}, C^{(k)}\right)}
$$

b) Clustering Dispersion Indicator (CDI):

$$
C D I=\frac{1}{d(R)} \sqrt{\frac{1}{K} \sum_{k=1}^{K} d^{2}\left(C^{(k)}\right)}
$$


The Mean Index Adequacy (MIA) depends on the average of the mean distances between each pattern assigned to the cluster and its center. The Clustering Dispersion Indicator (CDI) is directly proportional to the distance between the load diagrams in the same cluster and inversely proportional to the distance between the class representative load diagrams. The number of classes selected for our K-means algorithm must follow a balance between compactness and the purpose of the clustering. A reasonable number of classes will be between six and nine. Below six, the classes are too general while above nine the classes are too specific. The idea is to let electricity providers design the tariffs that will adapt to their consumers. Therefore, offering more than 6-7 different tariffs are too many as most of them only offer two currently, a fixed rate tariff and a Time-Of-Use rate tariff.

\section{Forecasting section}

The effectiveness of Gramian Angular Fields and Markov Transition Fields in classification problems become clear after the publication of "Encoding Time Series as Images for Visual Inspection and Classification Using Tiled Convolutional Neural Networks" by Zhiguang and Tim (2015). Wang's work considers the problem of encoding time series as images to allow machines to "visually" recognize, classify and learn structures and patterns. Here we summarized the three representations introduced by Zhiguang and Tim (2015). They are called Gramian Angular Summation/Difference Fields (GASF/GADF) and Markov Transition Fields (MTF). Table 4, shows an example of these three images for the representative profile of the last month of spring 2015. We generated these images using Faouzi (2018)

\section{Gramian Angular Field}

Gramian Angular Fields (GAF), proposed by Zhiguang and Tim (2015), are a way to encode angular information about the time series into an image. The image resulting from a GAF transformation is a quasi-Gramian matrix, computed on one of the two inner-product spaces,

$$
\begin{aligned}
& <x, y>=x \cdot y-\sqrt{1-x^{2}} \sqrt{1-y^{2}} \\
& <x, y>=y \cdot \sqrt{1-x^{2}}-x \cdot \sqrt{1-y^{2}}
\end{aligned}
$$

for Gramian Angular Summation Fields (GASF) and Gramian Angular Difference Fields (GADF) respectively. These Gramian matrices are equivalent to first normalize the time series data $X=\left\{x_{1}, x_{2}, \ldots, x_{n}\right\}$ to fall within the interval $[0,1]$ and convert them to polar coordinates such that $\phi_{n}=\arccos \left(x_{n}\right)$. After transforming the re-scaled time series into the polar coordinate system, it is easy to exploit the angular perspective by considering the trigonometric sum/difference between each point to identify the temporal correlation within different time intervals. The Gramian Angular Summation Field (GASF) and the
Gramian Angular Difference Field (GADF) are matrices whose elements are defined as follows:

$$
\begin{aligned}
G A S F_{i j} & =\left[\cos \left(\phi_{i}+\phi_{j}\right)\right] \\
G A D F_{i j} & =\left[\sin \left(\phi_{i}-\phi_{j}\right)\right]
\end{aligned}
$$

The encoding map explained by Wang and Oates has two important properties. First it is bijective as $\cos (\phi)$ is monotonic when $\phi \in[0, \pi]$. This means that given a time series, the proposed map produces one and only one result in the polar coordinate system with a unique inverse map. Therefore, a time series will have its unique GAF representation. It is important to re-scale the time series so that all values fall in the interval $[0,1]$ as this will permit to reconstruct the time series from the GASF as follows. The main diagonal of the GASF, i.e. $\left\{G_{i i}\right\}$ allow us to precisely reconstruct the original time series by:

$$
\cos (\phi)=\sqrt{\frac{\cos (2 \phi)+1}{2}}
$$

According to Zhiguang and Tim (2015), there are several advantages of GAF representation method: the bottom right element in the matrix contained the last information in the time series, and the old information may be placed on the upper and left parts. The timestamp of each data element in this matrix will be increased with rightward and downward axes. This attribute means that the generated graph can retain the temporal dependency, making the images without losing the property of the original data. GAFs give at least as much information as the time series itself with the elements of its diagonal. But it also gives additional information not available explicitly in the time series as the temporal correlations between elements separated a time period of $|i-j|=k$. Another import feature of this encoding map is that polar coordinates preserve absolute temporal relations. These features might have an important impact because the Neural Network might learn these feature in the training process.

\section{Markov Transition Field}

The MTFs encode dynamical transition probabilities sequentially to preserve information in the time domain. The steps to encode time series into MTFs can be summarized in three parts. First, identify the $\mathrm{Q}$ quantile bins of a time series and assign each $x_{i}$ to the corresponding bins $\mathrm{q}(q \in[1, Q])$. Then construct the Markov transition matrix $[W]=[Q x Q]$ by counting transitions among quantile bins. $\left\{W_{i i}\right\}$ accounts for self-transition probability whereas $\left\{W_{i j}\right\}$ shows the frequency with which a point in quantile $q_{j}$ follows a point in quantile $q_{i}$. Finally, the Markov Transition Field (MTF) is a $[N x N]$ matrix where $\mathrm{N}$ represents the number of elements of the time series. $\quad\left\{M_{i i}\right\}$ represents the self transition probability of the quantile $q_{i}$ at time $t_{i}$ whereas 
1-h vs 15-min Representative Spring Profile ID77
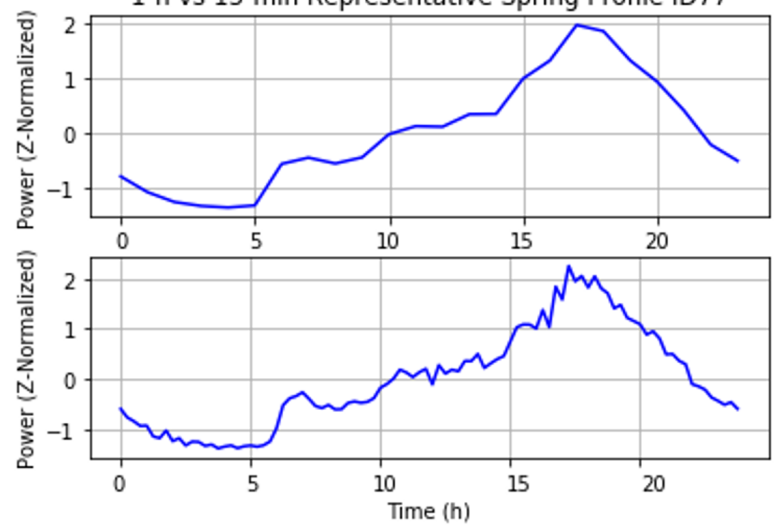

Figure 3: Comparison of the representative load profile of IDry for spring with a resolution of 1-hour and 15-min.

$\left\{M_{i j}\right\}$ represents the transition probability from the quantile at $t_{i}$ to the quantile at $t_{j}$. The map functions of MTF will produce one and only one image with fixed size and fixed number of quantiles $\mathrm{Q}$ for each given time series. Because its mapping functions are surjective, the inverse image of the mapping functions is not fixed, which means that it is not possible to recover the raw time series from its MTF.

Zhiguang and Tim (2015) stated that $G_{i j}$ denotes the superposition/difference of the directions at $t_{i}$ and $t_{j}$ whereas $M_{i j}$ is the transition probability from the quantile at $t_{i}$ to the quantile at $t_{j}$. GAF encodes static information because it contains temporal relations similarly to an autoregressive model (AR) while MTF depicts information about dynamics in a similar way to a logistic regression does. From this point of view, we consider them as three orthogonal channels, like different colors in the RGB image space. Thus, we can combine GAFs and MTF images of the same size (i.e. $S_{G A F s}=S_{M T F}$ ) to construct a triple-channel image (GASF-GADF-MTF).

\section{Simulation: case study on a data base of electric consumers}

In this case study, we focus on the months of spring and summer to give some significant results. The database contains information from 496 residential consumers between Texas, California and Colorado. This database is provided by PecanStreet (2018).

Following the procedures presented in the characterization section, we reduce and normalize the data. Each consumer is then described by a normalized representative daily load curve as Figure 3 illustrates. Figure 3 depicts the minimal differences between the representative load profile for the consumer ID77 depending on the sampling size.
Table 1: MIA and CDI of the summer and winter clusters depending on the number of clusters.

\begin{tabular}{|l|l|l|l|l|}
\hline \multicolumn{4}{|c|}{ Consumer characterization } \\
\hline & \multicolumn{2}{|c|}{ Spring } & \multicolumn{2}{c|}{ Summer } \\
\hline clusters & MIA & CDI & MIA & CDI \\
\hline 6 & 0.5637 & 0.9236 & 0.5555 & 0.9277 \\
7 & 0.5541 & 0.9335 & 0.5491 & 0.9471 \\
8 & 0.5402 & 0.9475 & 0.5260 & 0.9448 \\
9 & 0.5345 & 0.9246 & 0.5208 & 0.8776 \\
\hline
\end{tabular}

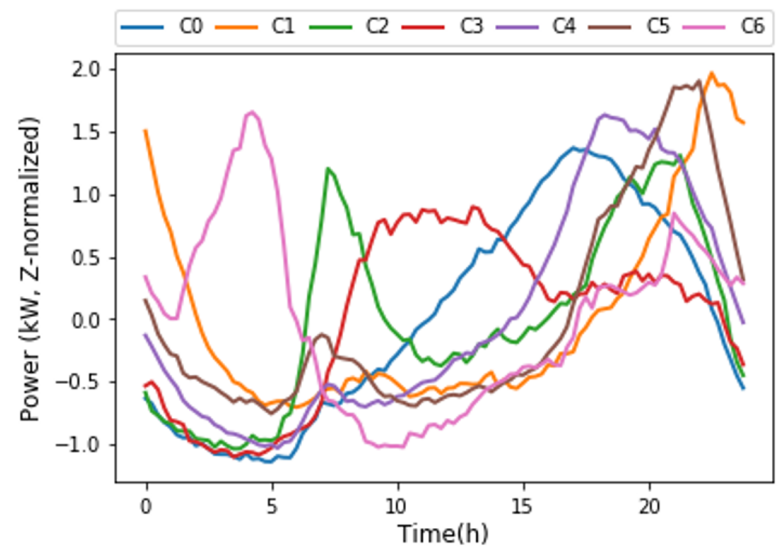

Figure 4: Cluster centroids for Spring 2015 workdays with the data collected in 15-min intervals.

Table 1 shows MIA and CDI compactness measures depending on the number of classes from six to nine. In general, as the number of classes increases, the MIA decreases while the CDI increases except for nine clusters. Nine classes seem to be the best answer regarding compactness, but it will make the forecasting task more challenging as we discuss later. Eight classes are even worse than nine because the clusters are less compact and eight classes are still challenging for the forecasting task. Seven classes was the final decision over six because an additional class will help in collecting all those consumers that have very particular profiles and are difficult to classify in other classes.

Figure 4 illustrates the cluster centroids for Spring 2015. At this point, all the consumer have been labeled from 0 to 6 depending on their spring consumption patterns. With a month of data, there is enough accuracy to classify new consumers in one of the classes that Figure 4 describes.

Due to the small size of the dataset (496 consumers), the accuracy of the neural network depends heavily on which elements were used for the training and which for the testing. Therefore, we trained the neural network model 20 different times to present its average test accuracy values and its standard deviation. The performance in the classification task of the K-means algorithm using the raw time 
Table 2: Classification results for Spring15 using the first month of Spring as input.

\begin{tabular}{|c|c|c|c|c|}
\hline Kmeans & \multicolumn{4}{|c|}{ CNNs } \\
\hline TS Accuracy & \multicolumn{2}{|c|}{ Test Accuracy } & \multicolumn{2}{c|}{ Train Accuracy } \\
\hline \multirow{2}{*}{$\mathbf{0 . 7 0 5 6}$} & $\mu$ & $\mathrm{s}$ & $\mu$ & $\mathrm{s}$ \\
\cline { 2 - 5 } & $\mathbf{0 . 6 4 6 4}$ & 0.0608 & 0.6523 & 0.0595 \\
\hline
\end{tabular}

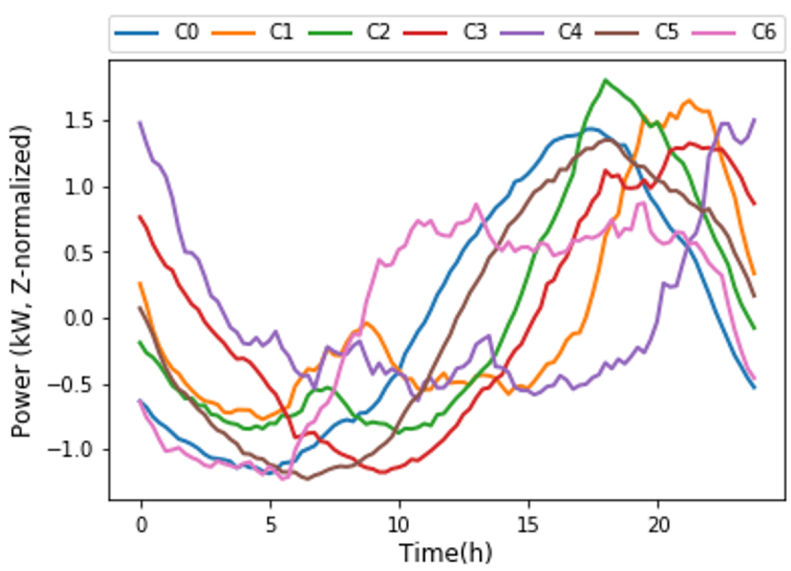

Figure 5: Cluster centroids for summer 2015 workdays with the data collected in 15-min intervals.

and the CNNs model using the MTFs and GAFs is compared in Table 2. In this case, Table 2 shows that the K-means classifier works better.

Figure 5 shows the different classes in summer. Cluster number 4 collects 15 elements with unusual behavior that could not be grouped in other clusters. These elements must be removed from the study. Figure 6 shows the representative daily profiles of consumer ID77 for the last month of Spring and for the whole summer while Table 4 shows the MTF, GASF, and GADF of the representative daily profile of the last month of spring 2015.

As we did in spring, two different classification
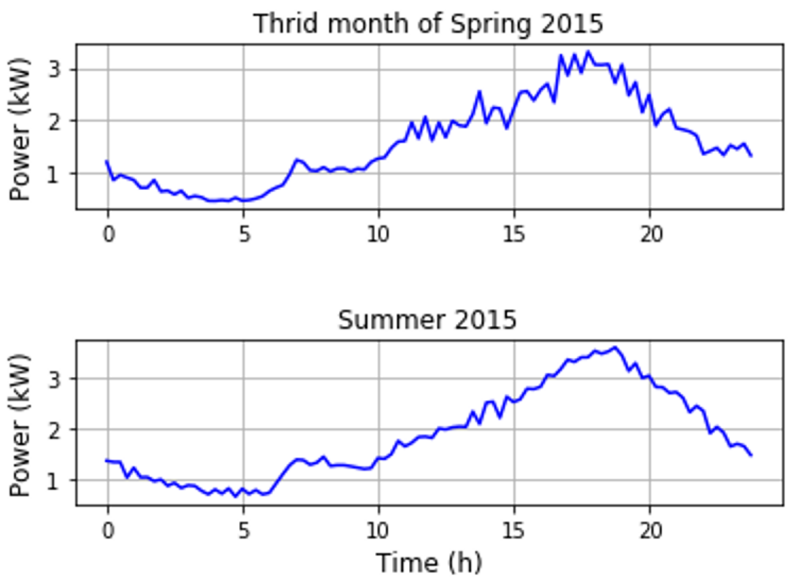

Figure 6: Comparison between the representative profile of the last month of spring and the three months of summer 2015 for consumer ID $7 \%$.
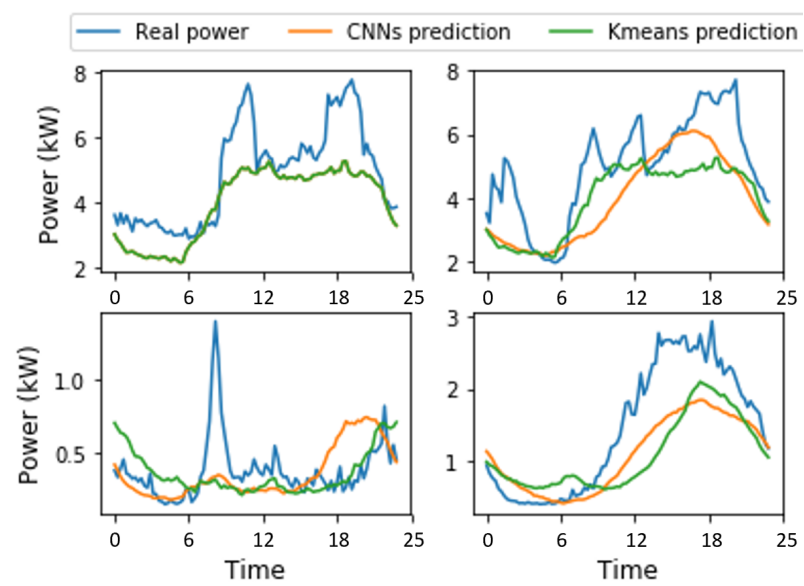

Figure 7: Comparison between the representative profile of the summer and the CNNs and K-means predictions using data only from spring for 4 consumers.

algorithms are compared in Table 3. In this case, the CNNs model performs significantly better than K-means algorithm, achieving around an 18\% improvement. From the results presented in Table 3, we can stated that the CNNs model is slightly overffited due to the reduce number of consumers used in the training.

Table 3: Classification results for summer 2015 using the last month of spring as input.

\begin{tabular}{|c|c|c|c|c|}
\hline Kmeans & \multicolumn{4}{|c|}{ CNNs } \\
\hline Accuracy & \multicolumn{2}{|c|}{ Test Accuracy } & \multicolumn{2}{c|}{ Train Accuracy } \\
\hline \multirow{2}{*}{$\mathbf{0 . 5 9 6 8}$} & $\mu$ & $\mathrm{s}$ & $\mu$ & $\mathrm{s}$ \\
\cline { 2 - 5 } & $\mathbf{0 . 7 0 2 3}$ & 0.0371 & 0.7330 & 0.0345 \\
\hline
\end{tabular}

Additionally, to measure the reduction of unpredictability in the demand, we have calculated different indicators to quantify the differences in power between the real values and the forecasts for summer 2015. The problem is that when we forecast summer classes, we loss information that is key to reconstruct the power profiles. In this case, we do not have the values for the mean and the standard deviation of each representative power profile that are used to denormalize the cluster centroids. We have the option of using the mean and standard deviation extracted from the last month of spring data. Figure 7 depicts the comparison between the real representative profiles and the predictions for 4 consumers. In the case in which CNNs and K-means agree in the same label, the profiles are overlapped. If we compute the difference along the same dimension (hour 0 , hour 1 , ...) between the real values and the predictions, we obtain a difference of the $22.33 \%$ for the K-means prediction and a difference of the $23.18 \%$ for the CNNs prediction.

On the other hand, if we had the real values of the average and the standard deviation the results would 

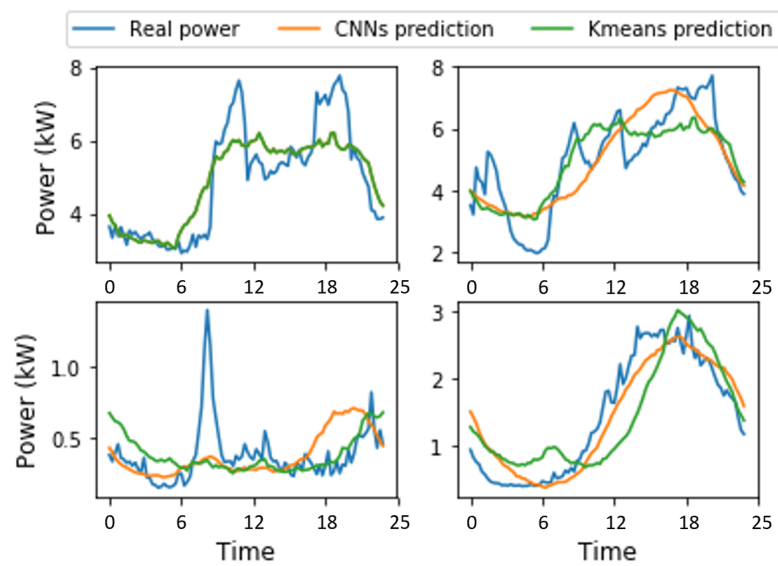

Figure 8: Comparison between the representative profile of the summer and the CNNs and K-means predictions using the real values of the average and standard deviation for 4 consumers.

be much more accurate. Figure 8 depicts the comparison between the real representative profiles and the predictions for 4 consumers using the real values of the average and standard deviation for summer. In this case the differences are significantly reduced reaching a $3.65 \%$ for the $\mathrm{K}$-means prediction and a difference of $0.65 \%$ for the CNNs prediction.

\section{Discussion and result analysis}

Regarding to the classification task, Table 5 shows the results of the spring classification depending on the numbers of classes with the objective of generalizing the results of the case study. Some variations were introduced to extract some relevant conclusions. For example, these variations include a K-means classification using the 3 channel images $(\mathrm{MTF}+\mathrm{GAFs})$ and using only the GAFs (2 channels). Raw TS means using the traditional time serie without transformations. In general, the K-means algorithm has a better performance than the CNNs. The reason of this is that the spring data have been labeled using a K-means algorithm and therefore the K-means algorithm for classification will give the best results possible. The K-means do not achieve an accuracy near to $100 \%$ because the labels have been created using the information of the three months of spring whereas the classification algorithm uses the data of the first month of spring. From Table 5 can be stated that CNNs have worse performance when the number of classes increases. This seems fair because the more classes are, the more difficult is for the CNNs to differentiate between very similar classes. Additionally, it appears that the MTFs have a smaller contribution to the K-means accuracy at all. The differences between using the three channels or using just the GAFs are very small. This is because the K-means algorithm better understands the GAFs than the MTFs. GAFs accurately represent
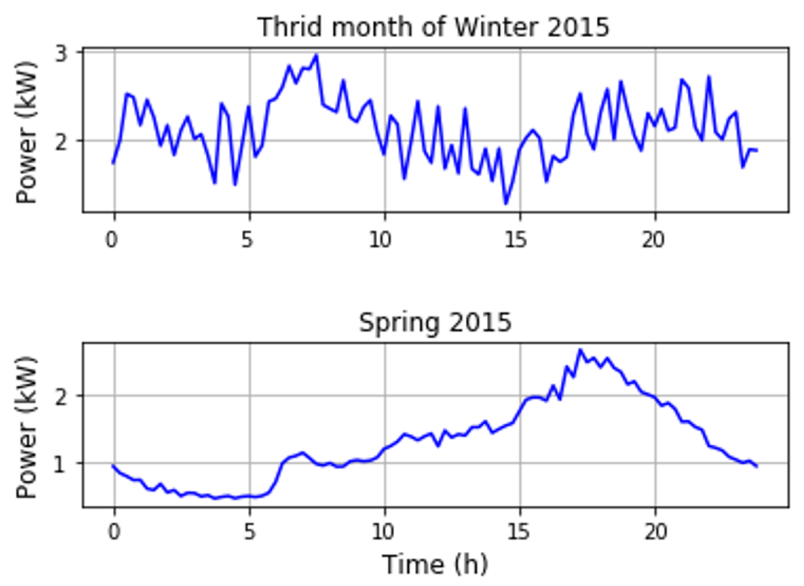

Figure 9: Comparison between the representative profile of the last month of winter and the Spring 2015 for consumer IDr7.

the time serie in a polar coordinate system while the MTFs provide information regarding transition probabilities in a time serie that the K-means algorithm does not know how to interpret.

With respect to the forecasting task, Table 6 shows the results of the summer forecasting depending on the numbers of classes with the objective of obtaining general results of the case study. It seems that the results obtained by the CNNs are significantly better. The more number of classes, the less accurate the forecasting algorithm is except for the CNNs model with 7 classes. Table 7 shows the results of forecasting every season of the year using the last month of the previous season to the one that is predicted. For this study we have used a reduced dataset of our initial data using only electricity consumers from Austin. This dataset gives us more compact clusters obtaining better results for the rest of the seasons. From the results, it seems that summer predictions are easier to generalize and work better for any dataset than the rest of the seasons. As we did for summer, the last month of winter tries to predict the classes of spring. It seems that a good or a bad performance has a lot to do with how similar are the time series of both periods. As it can be seen in Figure 9, the last month of Winter 2015 is very different than Spring 2015 while in Figure 6 the profiles are quite similar. This explains why the accuracy of forecasting summer is about a $70 \%$ while for spring is about a $38 \%$.

Regarding to Figure 7 and Figure 8, we can state that the most accurate way to measure the performance of our forecasts is using the 15-min power use differences as it gives us the differences of power in real-time. A good point to take into account is that consumers with higher power levels will have a bigger impact in our total energy results and therefore a bad classification of these elements will lead to worse results. 
Table 4: MTF, GASF, and GADF of the representative daily profile of the last month of spring 2015 for ID 77.
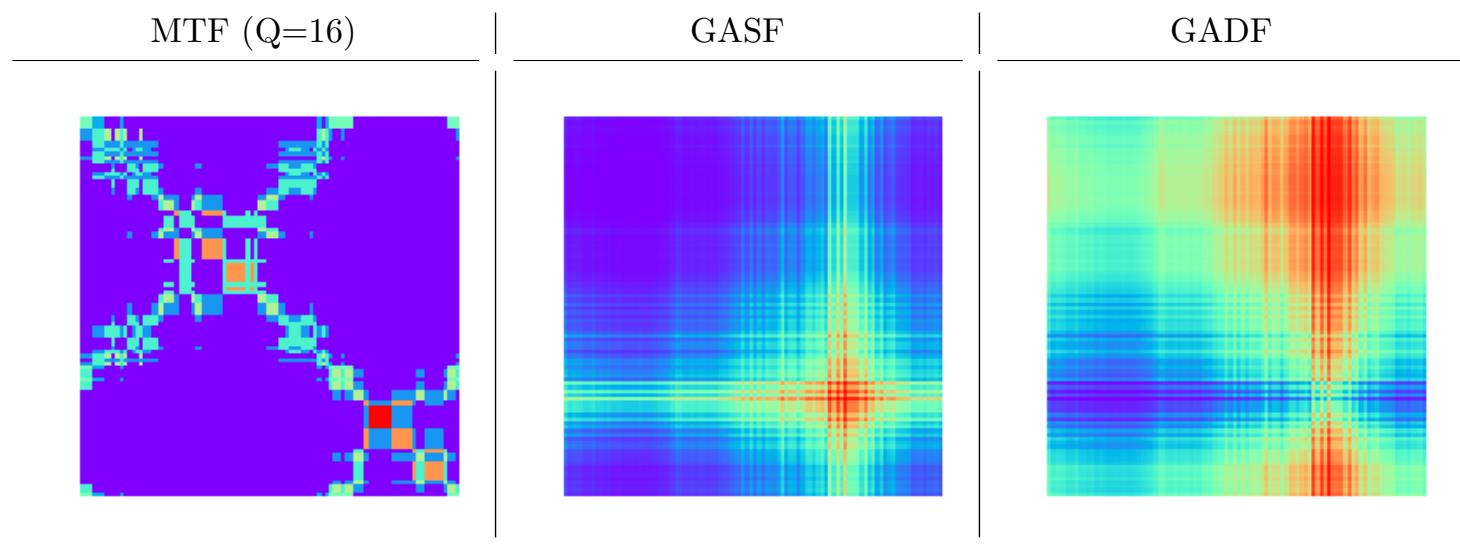

Table 5: Classification task results depending on the number of classes and the inputs used for K-means and CNNs.

\begin{tabular}{|c|c|c|c|c|c|c|c|}
\hline \multicolumn{9}{|c|}{ Spring 2015 Classification task } \\
\hline \multirow{2}{*}{$\begin{array}{c}\text { Number } \\
\text { of } \\
\text { Clusters }\end{array}$} & \multicolumn{3}{|c|}{$\begin{array}{c}\text { K-means } \\
\text { Accuracy }\end{array}$} & \multicolumn{2}{c|}{ Convolutional Neural Networks } \\
\cline { 2 - 8 } & Raw TS & GAFs & GAFs+MTF & $\mu$ & $\mathrm{s}$ & $\mu$ & $\mathrm{s}$ \\
\hline 6 & $\mathbf{0 . 7 0 9 6}$ & 0.6108 & 0.6189 & $\mathbf{0 . 7 1 7 1}$ & 0.0451 & 0.7330 & 0.0525 \\
7 & $\mathbf{0 . 7 0 5 6}$ & 0.5887 & 0.6108 & $\mathbf{0 . 6 4 6 4}$ & 0.0608 & 0.6523 & 0.0595 \\
8 & $\mathbf{0 . 6 9 9 5}$ & 0.5987 & 0.6230 & $\mathbf{0 . 6 9 6 9}$ & 0.0607 & 0.6973 & 0.0726 \\
9 & $\mathbf{0 . 6 6 7 3}$ & 0.5947 & 0.5987 & $\mathbf{0 . 6 3 6 3}$ & 0.0412 & 0.6398 & 0.0241 \\
\hline
\end{tabular}

Table 6: Summer 2015 Forecasting task results depending on the number of classes and the inputs used for K-means and CNNs.

\begin{tabular}{|c|c|c|c|c|c|c|c|}
\hline \multicolumn{9}{|c|}{ Summer 2015 Forecasting task } \\
\hline \multirow{2}{*}{$\begin{array}{c}\text { Number } \\
\text { of } \\
\text { Clusters }\end{array}$} & \multicolumn{3}{|c|}{$\begin{array}{c}\text { K-means } \\
\text { Accuracy }\end{array}$} & \multicolumn{3}{|c|}{ Tenvolutional Neural Networks } \\
\cline { 2 - 8 } & Raw TS & GAFs & GAFs+MTF & $\mu$ & $\mathrm{s}$ & $\mu$ & $\mathrm{S}$ \\
\hline 6 & $\mathbf{0 . 6 2 7 0}$ & 0.5423 & 0.5947 & $\mathbf{0 . 6 4 6 4}$ & 0.0439 & 0.6851 & 0.0387 \\
7 & $\mathbf{0 . 5 9 6 7}$ & 0.5242 & 0.5282 & $\mathbf{0 . 7 0 2 3}$ & 0.0371 & 0.7330 & 0.0345 \\
8 & $\mathbf{0 . 6 0 4 8}$ & 0.4879 & 0.5202 & $\mathbf{0 . 6 0 6 0}$ & 0.0755 & 0.6574 & 0.0240 \\
9 & $\mathbf{0 . 5 3 8 3}$ & 0.4697 & 0.4959 & $\mathbf{0 . 5 1 5 1}$ & 0.0521 & 0.5667 & 0.0241 \\
\hline
\end{tabular}

Table 7: Forecasting task results depending on the season of the year and using a reduced dataset with consumers from Austin only.

\begin{tabular}{|c|c|c|c|c|c|}
\hline \multicolumn{7}{|c|}{ Year 2015 Forecasting task } \\
\hline \multirow{3}{*}{ Season } & K-means & \multicolumn{2}{|c|}{ Convolutional Neural Networks } \\
\cline { 2 - 6 } & Accuracy & \multicolumn{2}{|c|}{ Test Accuracy } & \multicolumn{2}{|c|}{ Train Accuracy } \\
\cline { 2 - 6 } & Raw TS & $\mu$ & $\mathrm{s}$ & $\mu$ & $\mathrm{s}$ \\
\hline Summer & $\mathbf{0 . 6 3 6 8}$ & $\mathbf{0 . 6 0 8 0}$ & 0.4511 & 0.6830 & 0.0345 \\
Fall & $\mathbf{0 . 5 8 9 0}$ & $\mathbf{0 . 5 6 6 8}$ & 0.0371 & 0.7319 & 0.0586 \\
Winter & $\mathbf{0 . 5 3 9 8}$ & $\mathbf{0 . 5 2 0 5}$ & 0.0638 & 0.7072 & 0.0423 \\
Spring & $\mathbf{0 . 3 8 0 0}$ & $\mathbf{0 . 3 7 6 9}$ & 0.057 & 0.6761 & 0.0661 \\
\hline
\end{tabular}




\section{Conclusion}

In this work, we showed how state-of-the-art machine learning algorithms can be used in real-world applications. The high accuracy of CNNs models in classifying time series presented in other works was the inspiration to try these concepts in electrical demand forecasting. The definition of the GAFs and the MTF with the combination of Convolutional Neural Networks, gives new possibilities in time series analysis in a different way as it has been done traditionally. This work has covered how to extract significant information of load profiles shapes from large quantities of data and how to encode this data in images that can be used in classification tasks. This work also covers a procedure to use this data to generalize the typical electric consumers and to use this generalization to predict future demands. This forecasts will help to reduce the uncertainty in electric demand which will reduce the costs of electricity providers. The most significant point of this work is the high accuracy obtained in the prediction of future consumer behaviours. On the other hand, this works falls in obtaining an accurate clustering model that will correctly represent the general behaviour of our consumers. The next steps of our work, will be to prove these concepts in a real dataset provided by an electricity supplier to see how this procedure works and how it helps in tariff customization. Bigger datasets should give better results in terms of classification accuracy and consumer generalization.

\section{References}

Albadi, M. H. and E. F. El-Saadany (2007). Demand response in electricity markets: An overview. IEEE.

Chicco, G., R. Napoli, P. Postulache, M. Scutariu, and C. Toader (Feb 2003). Customer characterization options for improving the tariff offer. In Trans. Power Syst., vol. 18, no. 1, pp. 381-38\%. IEEE.

Faouzi, J. (2018, May). pyts: a Python package for time series transformation and classification. https://doi.org/10.5281/zenodo. 1244152. [Online; accessed May-2018].

Figueiredo, V., F. Duarte, F. Rodrigues, Z. Vale, C. Ramos, S. Ramos, and J. Gouveia (2003). Electric energy customer characterization by clustering. Greece.

Figueiredo, V., F. Rodrigues, Z. Vale, and J. Gouveia (2005). An electric energy consumer characterization framework based on data mining techniques.

Mill, S. (2016). Electric load forecasting: advantages and challenges. http://engineering. electrical-equipment.org. [Online; accessed January-2018].
PecanStreet (2018). Dataport. http://dataport. cloud. [Online; accessed January-2018].

Valero, S., M. Ortiz, F. Garcia, N. Encinas, A. Gabaldón, A. Molina, and E. Gómez (2004). Characterization and identification of electrical customers through the use of self-organizing maps and daily load parameters. IEEE.

Zhiguang, W. and O. Tim (2015). Encoding time series as images for visual inspection and classification using tiled convolutional neural networks. Association for the Advancement of Artificial Intelligence. 